\title{
INFORME LUZ DE LA SITUACIÓN DE LA EDUCACIÓN EN CHILE AL 2019 FORO POR EL DERECHO A LA EDUCACIÓN PÚBLICA
}

\section{REPORT ON THE STATE OF EDUCATION IN CHILE TO 2019 FORUM FOR THE RIGHT TO PUBLIC EDUCATION}

\author{
Juan González \\ Doctor en filosofía \\ Universidad de Chile \\ juangl@uchile.cl \\ Álvaro Carreño Sáez \\ Psicólogo-EPE \\ Universidad de Chile \\ alvarocs@zoho.com \\ Javier Figueroa Ortiz \\ Biólogo \\ ONG Entorno \\ oentorno@hotmail.com \\ Magaly Ávila Salinas \\ Comunicadora Social \\ Red de Periodistas y Comunicadores Migrantes \\ magallyavilasalinas@gmail.com \\ Consuelo Chaparro Gómez \\ Educadora Popular \\ CEEAL \\ consuelo.chaparro@yahoo.es \\ Diego Parra Moreno \\ Doctor en psicología \\ Universidad de Chile \\ diegoparram@gmail.com \\ Rodrigo Sánchez Edmonson \\ Licenciado en Historia \\ Universidad de Chile \\ rosanche@uchile.cl
}


Resumen: Este documento, en su versión en inglés, fue presentado en julio 2019 por el Foro pro el Derecho a la Educación Pública en el Hight Level Forum Political (HLFP) on Sustainable Development, en Nueva York. El HLPF es un mecanismo del sistema de las Naciones Unidas (ONU) para la rendición de cuentas, el seguimiento y la revisión de la Agenda 2030 de desarrollo sostenible y los Objetivos de Desarrollo Sostenible (ODS) a nivel mundial. Se trata de una plataforma en la que los gobiernos, sociedad civil y otros actores se reúnen para evaluar éxitos y dificultades en la implementación de los ODS en todo el mundo. Este Seminario fue organizado por la Campaña Mundial por la Educación (CME) y la Campaña Latinoamericana por el Derecho a la Educación (CLADE).

Palabras clave: derecho a la educación, objetivos de desarrollo sostenible, educación pública.

Abstract: This document was presented in July 2019 by the Forum for the Right to Public Education at the High Level Forum Political (HLFP) on Sustainable Development in New York. The HLPF is a United Nations (UN) system mechanism for accountability, monitoring and review of the 2030 Agenda for Sustainable Development and the Sustainable Development Goals (SDGs) at the global level. It is a platform where governments, civil society and other stakeholders come together to assess successes and challenges in the implementation of the SDGs around the world. This Seminar was organized by the Global Campaign for Education (GCE) and the Latin American Campaign for the Right to Education (CLADE).

Key words: right to education, sustainable development goals, public education.

\section{INTRODUCCIÓN}

El año 2015, el estado de Chile adhirió a la Declaración de Incheon, en el encuentro del Foro Mundial de Educación realizado en Corea del Sur, enmarcada en la agenda del Objetivo para el Desarrollo Sostenible número 4 (ODS4). En noviembre de ese mismo año la Asamblea General de Naciones Unidas adoptó una Agenda de Desarrollo para el año 2030, la que incluye en el Objetivo 4 (ODS4) el "Garantizar una educación inclusiva, equitativa y de calidad y promover oportunidades de aprendizaje a lo largo de toda la vida para todas y todos".

El análisis de lo sucedido en Chile hasta la fecha respecto de avanzar en el logro de ODS4 es regresivo, en tanto involucra las implicancias y el impacto del tsunami privatizador implementado en Chile hace más de 35 años, que se ve reafirmado con la marea reformista de los últimos 4 años, que han dejado a la educación pública en condiciones de desventaja, a la vez que ha fortalecido a sectores privados asociados a la oligarquía chilena.

Estos sectores tienen intereses en los distintos niveles del sistema educativo: parvulario, escolar y educación terciaria. Estos intereses se materializan de múltiples maneras; en el constante aumento de la matrícula en escuelas y en universidades privadas, el fortalecimiento financiero y jurídico de colegios y universidades privadas en propiedad de 
la elite, como también en la precarización de los servicios del Estado, y/o la penetración de las lógicas gerenciales de mercado en cada ámbito de la educación pública.

Aprovechamos esta oportunidad para denunciar que en Chile la educación pública se encuentra en una profunda crisis de sentido y de gestión en todos sus niveles educativos. La actual institucionalidad pública y los diversos gobiernos del país no han garantizado una educación inclusiva y equitativa de calidad ni han promovido oportunidades de aprendizaje permanente para todos. Asimismo, los gobiernos del país no han establecido marcos jurídicos y políticos que promuevan la rendición de cuentas y la transparencia, así como la dirección participativa y las asociaciones coordinadas en todos los niveles y todos los sectores, como tampoco han defendido el derecho a la participación de todas las partes, como se comprometió el estado de Chile al adherir a la Declaración de Incheon (2015).

Es en este marco, las organizaciones del Foro por el Derecho a la Educación Pública desarrollaremos los argumentos que demuestran el abandono en que el estado de Chile tiene al derecho de la educación a lo largo de toda la vida, Derecho Humano Fundamental y la base para garantizar la realización de otros derechos.

\section{CONTEXTO SOCIOECONÓMICO Y POLÍTICO DEL PAÍS}

Supuestamente, Chile es un país cercano al desarrollo, que integra la OCDE hace algunos años. Sin embargo, la desigualdad y la pobreza que sufre la sociedad chilena atenta con el derecho de la educación a lo largo de toda la vida para todas y todos.

A. La economía chilena ha mantenido un lento crecimiento en torno al $3 \%$ anual durante la última década. No obstante, la ciudadanía y organizaciones sociales han sido enfáticas en denunciar que dicho crecimiento económico sigue beneficiando a la elite y la oligarquía chilena, reafirmando la preocupante desigualdad en el país y sometiendo a un importante grupo de la población a una pobreza estructural permanente (Sáez, 2019). Actualmente el $20 \%$ de la población chilena sufre pobreza multifuncional. El 50\% de los hogares más pobres tenía en 2017 un 2,1\% de la riqueza del país, mientras que el 10\% más rico concentraba el 66,5\% (Comisión Económica para América Latina y el Caribe, 2019). Esta desigualdad se reproduce en el ámbito educativo. En Chile los años de escolaridad obligatorios son 13, la población de 15 años o más del decil de menores ingresos tiene en promedio 8,5 años de escolaridad frente a los 15,7 años que tiene el decil de mayores ingresos (Ministerio de Desarrollo Social, 2018). En términos de aprendizaje, esta desigualdad es evidente y clasista; todos los indicadores de calidad de la institucionalidad chilena muestran una correlación entre niveles de logro de los aprendizajes de las niñas y niños en edad escolar y la realidad socioeconómica de la familia. Esta situación se arrastra en el país por décadas y las tendencias no han sido modificadas por las políticas de los sucesivos gobiernos desde finales del siglo XX.

B. La estructura socioeconómica del país agrede el derecho a la educación y atenta contra los esperados avances en torno al ODS4, así lo ha manifestado reiteradamente el movimiento social por la educación. Las distintas políticas impulsadas, tanto en el 
gobierno de Michelle Bachelet como las que se han implementado en el actual gobierno de Sebastián Piñera, apuntan a desarticular la educación pública e incrementa la desigualdad, como se detallará en el presente informe.

C. Es necesario considerar también distintas características de la actual situación de Chile, tanto en aspectos económicos, institucionales y subjetivos. Un punto relevante es la alta tasa de endeudamiento de la población, que hasta el 2018 va en un aumento sostenido según el Banco Central, llegando al 73,3\% del ingreso disponible (Banco Central de Chile, 2018). Al profundizar lo anterior, nos encontramos con que un gran porcentaje de las personas en Chile se encuentran endeudadas, alrededor del $76 \%$, y esta deuda se debe principalmente a educación (por ejemplo, el Crédito con Aval del Estado para financiar la educación superior) y al consumo (compras en grandes tiendas o pagos del día a día) (CADEM, 2019). La privatización de la educación en Chile está excluyendo del sistema educativo a todos aquellos jóvenes que no tienen capacidad de endeudamiento. Un 18\% de las y los jóvenes entre 15 y 29 años ni estudia ni trabaja, y el 70\% de ellos ni siquiera está buscando trabajo (Carrillo, Espinoza, \& Valenzuela, 2018).

D. Otro aspecto relevante es la alta tasa de desaprobación que enfrenta la institucionalidad chilena en su más amplio espectro. Por ejemplo, la confianza en las Fuerzas Armadas y Carabineros (cuerpo policial) se encuentra en un 34\% y 32\%, respectivamente (CERC-MORI, 2019). Es necesario mencionar que desde hace años existe una fuerte crítica a estas instituciones, incluido su alto mando de generales, por estar envueltas en casos gravísimos de corrupción, desfalco y participación en hechos delictuales, especialmente Carabineros, quienes han sido los ejecutores de una represión sistemática a los movimientos sociales, incluyendo montajes para inculpar delitos inexistentes a dirigentes sociales, asesinatos y encubrimientos con apoyo del gobierno ${ }^{1}$. Los partidos políticos también han experimentado una enorme baja en la confianza por parte de la ciudadanía, situándose en un 5\%. El actual gobierno ha recibido una pésima evaluación por parte de la población, solo del $25 \%$, siendo el ámbito Educación uno de los tres peores evaluados, con una nota 3,5 de un máximo de 7 (Encuesta CEP, 2019). Por el contrario, los estudios de opinión pública sitúan tanto a los profesores de colegios como a los universitarios en los rangos más altos de evaluaciones de confianza por parte de la ciudadanía.

\section{DESARTICULACIÓN DE LA EDUCACIÓN PÚBLICA EN EL PAÍS}

A. Nos encontramos con un lamentable panorama de la educación pública en Chile: la matrícula en la educación municipal va en un declive sostenido a la vez que se fortalece al sector privado, en especial a los sostenedores que, por medio del

\footnotetext{
1 Para más información se recomienda leer los siguientes artículos: https://ciperchile.cl/2019/03/08/otrolunar-en-las-platas-del-ejercito-el-turno-de-los-millonarios-fondos-para-la-vivienda/; https://ciperchile.cl/pdfs/2017/abril/COMISION INVESTIGADORA CAMARA.pdf https://radio.uchile.cl/2018/06/20/pacogate-2-expertos-analizan-la-nueva-arista-del-fraude-al-interior-decarabineros/; https://ciperchile.cl/2019/02/01/muerte-de-catrillanca-asi-se-invento-la-version-falsa-decarabineros/
}

Departamento de Educación - Facultad de Ciencias Sociales - Universidad de Chile 
financiamiento compartido, han recibidos aportes de las familias y del Estado. Los establecimientos educacionales cubren solo un $34,8 \%$ de la matrícula del sistema escolar chileno.

\section{Figura 1}

Muestra la evolución de la matrícula en el sistema escolar chileno según dependencia de los establecimientos

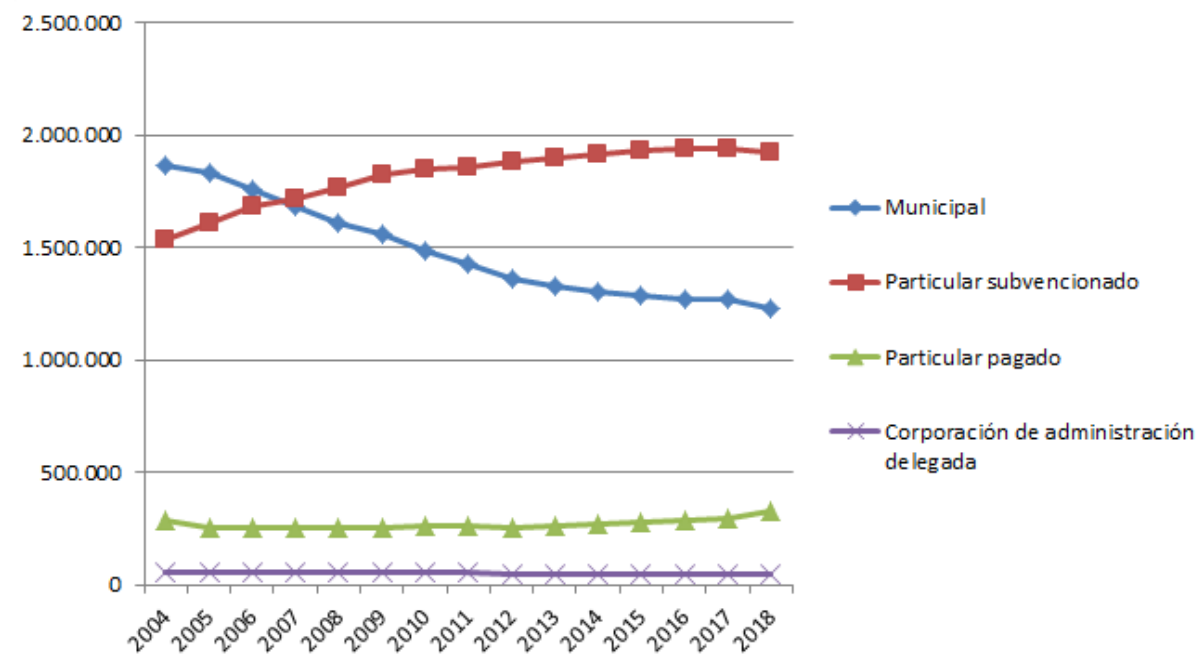

Fuente: Elaboración propia en base a datos estadísticos del MINEDUC.

B. La "Nueva Educación Pública" es un nuevo marco regulatorio aprobado por las diversas instancias legislativas de Chile en 2017. Esta reforma intentó legitimar los principios que sustentan el sistema subsidiario de la educación pública chilena, la protección de la educación privada y el concepto de la meritocracia especialmente en el acceso de la educación superior. Sin embargo, a menos de un año del inicio de su implementación tanto en la educación escolar como superior, las críticas ha sido lapidarias y no cuentan con el apoyo ciudadano. La reforma da continuidad a la lógica neoliberal del financiamiento por asistencia vía voucher o subsidio a la demanda, manteniendo un rol estatal débil y subsidiario. Además, promueve fenómenos de endoprivatización al hacer que las escuelas públicas e instituciones de educación superior estatales operen bajo lógicas gerenciales y economicistas, estableciendo indicadores y parámetros carentes de sentido pedagógico y propios de una visión que considera la educación como un bien de consumo y no un derecho humano.

C. El año 2011 se planteaba con fuerza, desde los amplios sectores movilizados en Chile, la consigna "educación pública, gratuita y de calidad" para darle un marco que cambiara el significado del derecho a la educación, en una línea distinta al paradigma de mercado imperante. El gobierno de Bachelet, presionada por el movimiento estudiantil y la ciudadanía, el año 2016implementó la llamada "gratuidad" para la 
educación superior, beneficio que abarcó en su primer período el arancel y matrícula de los estudiantes pertenecientes a los cinco primeros deciles de menores ingresos económicos que se matriculaban en alguna de las universidades elegibles (tanto públicas como privadas). Sin embargo, la denominada "gratuidad", no fue un beneficio universal ni permanente como fue exigido por la ciudadanía, transformándose en un subsidio a la demanda, que el estudiante puede perder si no cumple los requisitos establecidos por ley. En la actualidad, cerca de 27 mil estudiantes universitarios han perdido la gratuidad y cómo provienen de clases sociales sin recursos para endeudarse, ven con preocupación su futuro.

Tras la implementación de esta política, se evidencia que entre los presupuestos 2015 y 2016 hubo una disminución del 51,6\% de los recursos para becas de arancel y aumentó en un 37\% los recursos para el cuestionado Crédito con Aval del Estado $(\mathrm{CAE})^{2}$, lo que dejó en una precaria situación y susceptibles de endeudarse a estudiantes que no lograban cumplir con los requisitos de la "gratuidad" (Fundación Nodo XXI, 2016). La falta de control sobre aspectos como las alzas de aranceles o un aumento de matrícula por parte de las entidades privadas, que obtienen por vía de esta política recursos estatales, permite constatar que en la práctica opera de forma similar a un voucher que, unido con la prueba de ingreso a las universidades (PSU), genera el efecto de segregar aún más la educación superior en Chile y fortalecer los grandes consorcios privados de la educación superior.

Actualmente se discute en el parlamento una ley para extender el subsidio a la demanda a la educación parvularia. El proyecto de ley de subvenciones a los niveles sociales medios generó la oposición de asociaciones de trabajadoras/es, investigadoras/es y académicos de la educación inicial, que hacen ver el peligro de reproducir los efectos que este modelo ha generado en el sistema de educación escolar obligatoria y superior (Andrade, 2019).

D. En general, las políticas implementadas no han contado con la participación activa de los actores involucrados en el sistema educativo, muy por el contrario, han sido generadas a través del lobby y a espaldas de los movimientos sociales. Por ejemplo, todos los cambios curriculares en Chile han sido elaborados y ejecutados en forma inconsulta desde el Ministerio de Educación, imponiendo un currículum descontextualizado y sin considerar a las diversas naciones que habitan el territorio chileno (Mapuche, Aymara, Rapanui, etc.). Últimamente, los docentes han cuestionado modificaciones curriculares inconsultas, que fueron aprobadas por un órgano político superior, denominado Consejo Nacional de Educación, o el proyecto llamado por el gobierno como "Admisión Justa" que busca ampliar el porcentaje de estudiantes que pueden ser seleccionados por los establecimientos escolares, promoviendo aún más

\footnotetext{
${ }^{2}$ La expansión de la educación superior privada a partir del CAE aumentó El año 2005 (antes de que comience a operar el CAE), se registra una matrícula total de 663.679 alumnos, mientras el año 2017 se llega a 1.247.746 matriculados. Sin embargo, el 70\% de este aumento se concentra en sólo 20 instituciones que pertenecen a los siguientes grupos educacionales: i) Laureate International; ii) Santo Tomás; iii) INACAP y iv) Pontificia Universidad Católica. Por otro lado, en este período, la matrícula de los establecimientos estatales sólo aumenta 23.358 alumnos y su participación en el total se reduce a sólo 15,6\%, .Fundación SOL, 2018. Endeudar para gobernar y mercantilizar: El caso del CAE. Disponible en http://www.fundacionsol.cl/estudios/cae2018/
} 
la segregación e inequidad educativa y protegiendo de sobremanera los intereses de los colegios de la elite (Foro por el derecho a la educación pública, 2019).

\section{SENTIDO DE LA EDUCACIÓN}

A. La Declaración Universal de Derechos Humanos señala que en su artículo 26 que la educación tendrá por objeto el pleno desarrollo humano y el fortalecimiento del respeto a los derechos humanos, lo que se encuentra en consonancia con la meta 4.7 del ODS4 que establece entre otros puntos la educación para el desarrollo sustentable y los derechos humanos, a la vez que se promueve una cultura de paz y no violencia y la valoración de la diversidad cultural. Estos son puntos fundamentales en los desafíos educativos que enfrenta Chile, pero que actualmente presentan en el país una serie de falencias y deudas.

B. Los análisis que apuntan a que el sistema educacional chileno tiene una crisis de sentido no son nuevos. Ya en el contexto de las movilizaciones del año 2006 se discutía sobre el tipo de individuos que formaba esta sociedad al tener como valor central al mercado; sujetos consumidores, competitivos e individualistas (Colegio de Profesores de Chile A.G., 2006) permeando el currículum y el sistema educativo, reflexiones que siguen totalmente vigentes hasta hoy, demostrando que son aspectos estructurales del modelo chileno. Lo demuestra el estancamiento en los aprendizajes, particularmente en los sectores populares (OECD, 2018).

C. La educación en Chile no apunta a lograr el máximo desarrollo posible de los estudiantes en su conjunto, al existir una alta segregación influida directamente por la capacidad de pago de las familias y su estatus socioeconómico. Lo máximo que pueden aspirar muchos de los hijos de las clases trabajadoras y populares es terminar con éxito la educación secundaria. Un grupo minoritario ingresa a la educación superior y de ellos, un sector muy marginal puede finalmente egresar de una carrera profesional. En los conceptos ideológicos de las clases dominantes en Chile, la educación superior no es considerada un derecho sino el resultado del mérito del estudiante a lo largo de la enseñanza escolar. El resultado de esta concepción clasista y elitista es la fuerte desigualdad económica y social en el sistema educacional chileno que posteriormente se traduce en el mercado laboral y en el ámbito sociocultural. ${ }^{3}$

D. Las lógicas homogeneizadoras están instaladas en el sistema educativo chileno, principalmente a través de las pruebas SIMCE (Sistema de Medición de la Calidad de la Educación) y PSU (Prueba de Selección Universitaria), estancados en los últimos años y con cada vez más consistente crítica de distintos actores educativos respecto a ellas. Incluso organizaciones de estudiantes han realizado actos de resistencia y

\footnotetext{
3 Peor aún, en Chile la elite se reproduce a sí misma. Nada más lejos del mérito académico y/o laboral. "El economista de Yale, Seth Zimmerman, mostró en 2013 que el 50\% de los cargos más altos en las empresas chilenas lo ocupan ex alumnos de sólo nueve colegios de elite. Para el economista Ricardo Hausmann la tendencia de que los mejores puestos queden en manos de personas de un mismo origen, da cuenta de una cultura empresarial cerrada, que no da oportunidades a talentosos de otras clases". Ver en: “Cómo la elite nos hace creer que triunfa porque es inteligente y trabajadora". Disponible en: https://ciperchile.cl/2018/07/17/como-la-elite-nos-hace-creer-que-triunfa-porque-es-inteligente-ytrabajadora/
}

Departamento de Educación - Facultad de Ciencias Sociales - Universidad de Chile 
denuncia- no rendir el SIMCE cuando es aplicado en sus escuelas. Estas evaluaciones han sido ampliamente criticadas porque sus resultados dependen fuertemente de las condiciones socioeconómicas de las familias de los estudiantes y no reflejan sus procesos de aprendizaje. Respecto al SIMCE se ha señalado: "Esta prueba censal ha ido ampliando progresivamente sus grados y materias, constriñendo el currículo, incentivando los ensayos de preparación, desprofesionalizando el trabajo docente, aumentando el agobio laboral y el estrés en la comunidad escolar" (Inzunza \& Campos-Martínez, 2016) ${ }^{4}$. Asimismo, la propia existencia de la prueba de selección universitaria (PSU) reconoce implícitamente todas las deficiencias en la calidad de la educación secundaria, ya que los estudiantes no pueden rendir con éxito estas pruebas sino son preparadas por años en instituciones privadas extra-escolares con altos costos materiales y emocionales para los jóvenes y sus familias. Nuevamente se demuestra que la meritocracia es un fenómeno ilusorio, simplemente esconde con un velo atractivo el clasismo y elitismo de la sociedad chilena.

\section{Educación Inclusiva}

A. La inclusión en el sistema educativo es uno de los grandes desafíos que están pendientes para las y los trabajadores y el pueblo de Chile. Así lo establece en términos generales el informe internacional Right to Education Index del año 2018, que señala la falta de acciones el pos de la inclusión de la gran diversidad de estudiantes presentes en el sistema educativo en Chile que, con su gran estandarización y normalización, impide que los hijos de trabajadores, como indígenas, migrantes y otras minorías marginadas por la oligarquía y la elite chilena, cuenten con espacios adecuados a sus necesidades.

B. En los últimos años, uno de los hitos en torno a la inclusión fue en mayo de 2015, cuando se promulgó en Chile la Ley de Inclusión Escolar, un complejo conjunto de artículos y modificaciones a cuerpos legales preexistentes, que planteaban como uno de sus fundamentos, el fortalecimiento de la educación pública, teniendo como uno de sus pilares el fin al lucro, garantizando que los recursos económicos sean destinados a fines educativos; el fin al copago, aumentando los recursos estatales y disminuyendo los realizados por las familias; y la regulación de la admisión escolar, creando un sistema único y centralizado de admisión. Esta ley, que abarcaba múltiples reformas, recibió fuertes críticas del movimiento social, como que no realizaba cambios estructurales al sistema educativo manteniendo la preponderancia jurídica de la libertad de enseñanza por sobre el derecho a la educación, potenciando la provisión privada al aumentar la subvención sin modificar la forma en la que se entregan los recursos. La modificación es que los sostenedores recibirían los recursos directamente del Estado, en lugar de que los reciban por medio del copago de las

\footnotetext{
${ }^{4}$ También se recomienda la revisión del informe de María Teresa Flórez, 2013. Análisis crítico de la validez del Sistema de Medición de la Calidad de la Educación (SIMCE). Disponible en: http://www.opech.cl/wp/wpcontent/uploads/2017/10/informe-simce-oxford.pdf
} 
familias. Actualmente, el financiamiento compartido continúa vigente en las escuelas hasta el 2030, criticándose también la inexistencia de mecanismos certeros que aseguren el fin al lucro y lo difuso de los plazos para lograr dicho objetivo (Foro Chileno por el Derecho a la Educación, 2015)·Por el contrario, se normalizaría el lucro o la extracción de ganancias por parte de otros privados a partir de recursos estatales para la mejora educativa, alimentación, textos escolares, evaluación, investigación y acceso, entre otras áreas. La reforma en cuestión "no excluye a nadie del sistema educativo. Suma a bancos, grupos empresariales, empresas varias, en la gestión total de un sistema de provisión mixta, con un fuerte pilar privado, que se consolida bajo el control de los grupos de poder e inversionistas" (González \& Parra, 2016). De esta forma se constituye una profundización de las políticas impulsadas desde la dictadura militar tendientes a la privatización y a la desarticulación de la educación pública.

Uno de los aspectos que más llamó la atención de la ley fue en lo referente a acabar con los mecanismos de selección de las escuelas ${ }^{5}$, y que potencialmente podría promover la inclusión social en las escuelas. Sin embargo, se mantuvieron ciertos porcentajes de selección para determinados establecimientos. Además, la medida se ha aplazado en varios años, en base a reglamentos que no se han elaborado. Incluso esta medida está siendo cuestionada por el actual gobierno de Sebastián Piñera, con el proyecto de ley de "Admisión Justa" intentando aumentar los porcentajes de selección de los estudiantes a los establecimientos educativos.

C. Otro de los sucesos que ha marcado la agenda educacional en Chile durante los últimos meses es la implementación de la Ley Aula Segura que, amparada mediáticamente en el "combate a la violencia en las escuelas", ha dado un duro golpe a las comunidades educativas y a su democracia interna, atentando seriamente contra el ODS4, específicamente contra la inclusión escolar y las oportunidades de aprendizaje para todos. Esta ley, obviando cualquier análisis sobre la violencia estructural presente en el sistema educativo, otorga al director de un establecimiento educacional antes de un debido proceso la facultad de suspender o cancelar la matrícula a estudiantes que cometan actos que atenten contra la convivencia escolar, que pueden ser fácilmente utilizadas para reprimir las protestas estudiantiles. Existen dos problemas graves: Primero, no se garantiza el derecho a la educación de los estudiantes que sufran estas medidas, planteando sanciones de carácter netamente punitivo y criminalizante; segundo, los criterios para determinar qué acciones son punibles y sus sanciones descansa solamente en el criterio del director y en el reglamento interno del establecimiento educacional, documentos ampliamente cuestionados tanto por no ser construidos inclusiva ni democráticamente por las comunidades educativas, como por

\footnotetext{
${ }^{5}$ La selección de estudiantes ha sido históricamente defendida por la iglesia y amplios sectores de la derecha empresarial ligada al ámbito educativo. Incluye prácticas discriminatorias, como condicionar la matrícula en una escuela a elementos como las habilidades del niño o niña, las características de su familia y su credo religioso. Se puede profundizar en este tema en los siguientes documentos del Observatorio Chileno de Políticas Educativas: Cuando la selección de alumnos se convierte en discriminación. Disponible en: http://www.opech.cl/editoriales/2007 03/seleccion alumnos.pdf

¿Reforma y educacional? Sobre la nueva ley que regula el lucro, copago y selección. Disponible en: http://www.opech.cl/wp/wp-content/uploads/2015/08/Reforma-y-educacional-sobre-la-nueva-ley-queregula-el-lucro-copago-y-selecci\%C3\%B3n.pdf
} 
ser de plano ilegales al omitir o transgredir derechos fundamentales de los estudiantes, según UNICEF6.

A esta ley se suma el hecho de que las fuerzas policiales irrumpen cotidianamente en colegios movilizados, golpeando y encarcelando a estudiantes. El clímax del conflicto se vive actualmente en el Instituto Nacional, colegio de Santiago, donde la ministra de Educación y el sostenedor del colegio están enfocados en derrotar al movimiento estudiantil aunque sus acciones signifiquen costos físicos y emocionales para niños y adolescentes y la comunidad educativa ${ }^{7}$. En este punto, hacemos un llamado a los organismos internacionales pertinentes para que inste al gobierno de Chile a que abandone su política de terror y represión contra niñas y niños y deje de considerar a los dirigentes estudiantiles como "manzanas podridas ${ }^{8}$ y los considere como la comunidad civilizada internacional señala, niños y adolescentes que viven una etapa importante de su formación personal. El Foro por el Derecho a la Educación Pública ha debido constituir una red de organizaciones contra la represión de estudiantes, con el fin de instar al Estado a detener estas acciones de persistente criminalización del movimiento estudiantil (Diario UChile, 2019).

D. Formalmente, las leyes en Chile dicen promover la inclusión en el sistema educativo, tanto de estudiantes embarazadas, cómo de aquellas y aquellos que presenten alguna Necesidad Educativa Especial (NEE). Sin embargo, en la práctica estas políticas van de la mano con una tendencia a la privatización y precarización educativa, por ejemplo, al entregar una mayor subvención, que fluctúa históricamente entre el doble y el triple de la basal, a escuelas que incorporen a estudiantes con alguna NEE, generando así un "incentivo perverso" para su sobre diagnóstico. Un aspecto a considerar es que aunque en términos generales ha existido una tendencia al aumento de la cobertura en la educación secundaria, aparece un serio problema al analizarla, como la gran cantidad de estudiantes que abandonan o quedan excluidos del sistema escolar en lo que constituye una cifra poco transparente que solo recientemente se ha comenzado a analizar y que estimaría en 360 mil jóvenes que no asisten a clases, 2,6 veces más que los datos anteriores. ${ }^{9}$ Muchos de estos jóvenes que abandonan la escuela terminan en la delincuencia, el narcotráfico y la desesperanza.

E. No ha habido mayores avances durante los últimos años en el reconocimiento e inclusión de los pueblos originarios del país. Aunque formalmente se fomente su integración, la estructura rígida del sistema educativo impide hacer modificaciones

\footnotetext{
${ }^{6}$ Se puede profundizar sobre este tema en el siguiente artículo: La violencia en la escuela pública y la "reglamentarización" de la convivencia escolar. Disponible en: https://www.opech.cl/la-violencia-en-laescuela-publica-y-la-reglamentarizacion-de-la-convivencia-escolar/

${ }^{7}$ Para información sobre este tema actual que están viviendo las comunidades educativas de la comuna de Santiago ver página web del Foro: http://derechoeducacionpublica.cl/category/noticias/nacionales/

8 Ver noticia sobre esto: https://www.eldinamo.cl/actualidad/2018/10/13/alessandri-pide-sacar-lasmanzanas-podridas-de-los-liceos-y-ex-actor-de-mega-le-responde-sin-filtro/

${ }^{9}$ Se puede profundizar sobre los múltiples aspectos de esta temática en el siguiente reportaje: "Menores que abandonan la escuela: fondos públicos para colegios de reinserción cayeron en $\$ 1.300$ millones". Disponible en: https://ciperchile.cl/2019/03/19/menores-que-abandonan-la-escuela-fondos-publicos-para-colegios-dereinsercion-cayeron-en-1-300-millones/
} 
para lograr una educación pertinente y diversa. Esta inclusión se vuelve especialmente compleja de abordar cuando el Estado reprime especial y sistemáticamente al pueblo mapuche, vulnerando los derechos fundamentales de niños y niñas, víctimas directas de la violencia policial (El Desconcierto, 2017).Un caso de represión brutal con consecuencia de muerte fue el acribillamiento del dirigente mapuche Camilo Catrillanca en el año 2018, cuando un operativo dirigido directamente por el Ministerio del Interior del gobierno de Sebastián Piñera y del Alto Mando de Carabineros irrumpió en su comunidad, dándole muerte de manera instantánea después de cientos de balazos cuando regresaba a su hogar en un tractor desde su trabajo diario. En 2011, Camilo Cantrillanca era un dirigente estudiantil reconocido en su colegio, luchó y se movilizó muchas veces en su región para exigir una educación equitativa y de calidad. El gobierno de Chile lo terminó castigando con su don más preciado, su vida, aunque en las tierras de sus ancestros y descendientes seguirá viva su dignidad. ${ }^{10}$

F. Aunque culturalmente se ha avanzado en la disminución de las brechas de género en Chile, siguen existiendo importantes desafíos: las mujeres tienen en promedio menos años de escolaridad, más desocupación y reciben menos ingresos por el mismo trabajo y teniendo los mismos años de escolaridad que los hombres. Esta diferencia es más notoria mientras más años de escolaridad tengan. Por ejemplo, con 18 o más años de escolaridad, el ingreso promedio por la ocupación principal de las mujeres al año 2017 era de $\$ 1.259 .195$, mientras que para los hombres era de $\$ 1.969 .1372$.

La institucionalidad chilena aún cobija colegios públicos y privados unigenéricos, solo de niñas o solo de niños, incrementando la segregación por género y por sexo. Algunas comunidades escolares, presionadas por los jóvenes que entienden que la educación debe ser integradora, han optado por ser mixtos. Lamentablemente esta no es una política de la institucionalidad educativa del país y a la fecha la mayoría de los colegios tradicionales continúan siendo unigenéricos.

G. En cuanto a la inclusión educativa de la comunidad LGBTIQ+, nuestro país está dando pequeños pasos. En 2017, a nivel institucional, se publicó una Circular de Derechos y "Orientaciones para la Inclusión de Personas lesbianas, gays, bisexuales, trans e intersex", por parte del MINEDUC (Ministerio de Educación). También las nuevas generaciones de estudiantes son mucho más respetuosas con la diversidad.

Sin embargo, existen enormes desafíos que afrontar. Un 76,1\% de la población trans dice que ha sufrido discriminación en razón de su identidad de género, un 22,2\% sindica como responsables de los abusos a compañeros de estudios y un $19,9 \%$ a un docente. Un 15,4\% no expresa su género por temor a recibir malos tratos en su lugar de estudios (MOVILH, 2018). El principal momento de la vida en que personas trans reciben daño y dolor es en la infancia y adolescencia, ligado estrechamente al ámbito escolar, por lo que es importante que se tomen medidas efectivas que garanticen sus

\footnotetext{
10 Ver video Camilo Catrillanca Dirigente estudiantil. Toma Municipalidad Ercilla Mapuche Estudiantes.
} Disponible en: https://www.youtube.com/watch?v=UFRQaqifnNw\&t=30s 
derechos y bienestar físico y emocional, potenciando también la investigación y recopilación de información estadística al respecto por parte del Estado ya que es muy precaria.

\section{EDUCACIÓN A LO LARGO DE LA VIDA}

A. En Chile, la educación parvularia es el nivel más deficitario del sistema. Contempla distintos niveles que van desde los 0 hasta los 6 años de edad, partiendo en la sala cuna y terminando en el segundo nivel de transición, antes de entrar a la educación básica. $\mathrm{Al}$ año 2018, la matrícula total ascendía a 800.083 niños, quedando una gran parte sin acceso a la educación, correspondiendo a una cobertura general del $54,8 \%$, fuertemente concentrada entre los 4 y 5 años y alcanzando solo al $20,1 \%$ de quienes tienen entre 0 y 2 años.

La provisión en la educación parvularia contempla la participación de entidades públicas y privadas, marcado por una fuerte presencia de estas últimas con casi el $60 \%$ de la matrícula total (Subsecretaría de Educación Parvularia, 2019). Es necesario comprender la importancia de frenar en esta etapa escolar la enorme segregación existente, a la vez que se construye una educación pertinente a las necesidades de niños y niñas, considerándolos así en su momento actual y no desde una óptica adultocéntrica, mucho menos propiciando un estrechamiento curricular por medio del énfasis en contenidos (lenguaje y matemáticas) que serán de utilidad para su posterior evaluación dentro de un sistema que tiende a la estandarización y homogeneización.

B) El actual gobierno está promoviendo cambios en la legislación de la educación parvularia. Existen críticas a las nuevas propuestas, principalmente desde las educadoras, ya que sus medidas podrían precarizar y privatizar aún más la educación parvularia, a través del establecimiento de un sistema de subvenciones por parte del Estado, sin garantizar un financiamiento basal y aumentando el desapego familiar por las largas horas que los párvulos permanecerían en los jardines infantiles (Espacio Vinculante por la Nueva Educación Pública, 2019). El establecer un financiamiento por asistencia a las instituciones que imparten la educación parvularia, teniendo como evidencia que existe una diferencia concreta en la tasa de asistencia entre el primer y el quinto quintil, un $45,3 \%$ y un $57,8 \%$ respectivamente, es decir, niños y niñas de una mejor situación económica tienen una mayor asistencia, significa estar entregando más recursos para la educación de un sector determinado y privilegiado de la población, que provoca un aumento de la segregación y exclusión en los niveles preescolares del sistema educativo.

C) Respecto a la educación secundaria no hay avances respecto a los logros del ODS4, está pendiente el desafío de potenciar la democracia interna en las escuelas y en el sistema educativo en su conjunto. En general, los estudiantes no tienen participación en la elaboración de políticas ni en la toma de decisiones, muy por el contrario, las nuevas políticas suelen ser elaboradas a sus espaldas y aprobadas por el Congreso 
durante las vacaciones estudiantiles, dificultando que estudiantes y comunidades educativas puedan responder. Asimismo, los docentes se han precarizado y transformado en simples replicadores de programas y actividades elaboradas desde las oficinas centrales, con muy pocos espacios y tiempos para innovar durante el proceso educativo. Esta tendencia en la forma de decidir sobre políticas educativas se ve reflejada en la implementación de las nuevas políticas mencionadas anteriormente en este informe, tendientes a otorgar mayor poder en la gestión y facultades punitivas a los directores de establecimientos educacionales.

D. La reforma constitucional del año 2002 estableció que la Enseñanza secundaria fuera obligatoria y con opciones gratuitas. Se comenzó a desarrollar la Educación de Personas Jóvenes y Adultas (EPJA) buscando la finalización de estudios básicos o medios, sumándose luego iniciativas para lograr una alfabetización básica y contar con alternativas educativas en contextos de encierro (carcelario o de reclusión de menores de edad). Sin embargo, la eficacia de estos programas es cuestionable; por una parte, quienes más han participado son personas entre 14 y 18 años, en mayor proporción que durante años anteriores, lo que indica que personas de más edad están dejando de participar.

Aunque las cifras sobre escolarización en Chile muestran un aumento en los años de escolaridad con el paso del tiempo, existen complejas realidades tras ellos, como las que muestran los últimos datos de la Encuesta de Caracterización Socioeconómica Nacional(CASEN) de 2017: respecto al máximo nivel educacional alcanzado, señala que el 5,2\% de la población entre 30 a 44 años de edad no ha completado su Educación Básica y el 8,8\% su Educación Media; es peor la situación en tramos de más edad, como el de 45 a 59 años, el 14,7\% de ellos no ha completado su Educación Básica, y un 13,2\% su Educación Media. Es urgente volver a plantearse la población objetivo de estos programas, y preguntarse por qué una parte considerable de la población adulta en Chile aún no logra completar su educación obligatoria, que debería estar garantizada por el Estado. La educación a lo largo de la vida es una deuda del sistema educativo en Chile.

\section{FINANCIAMIENTO DE LA EDUCACIÓN}

A. El gasto público en educación ha aumentado en los últimos años, el dinero que recibirá el MINEDUC para el año 2019 representa un aumento del 2,7\% respecto al año anterior. No obstante, el gasto en educación como porcentaje del PIB el 2016 fue del $5,35 \%$ (Banco Mundial) y no llega al 7\% que recomienda UNESCO.

B. En Chile, en el sistema escolar se financia con la misma preferencia la educación pública y la particular subvencionada y esto se realiza por medio de una subvención básica por estudiante que asiste a clases, lo que genera una competencia entre las escuelas por captar matrícula (Cornejo, 2006). 
C. Como se analizó en el Eje 2, Chile se ha caracterizado por un aumento sostenido de la participación de entidades privadas en detrimento de las públicas, lo que ha generado el surgimiento de distintos tipos de sostenedores que buscan el lucro con los recursos que perciben de las familias y del Estado, existiendo incluso holdings educacionales organizados para tal efecto.

La consecuencia directa es que, si las condiciones de base del sistema educativo son una mayoritaria participación de privados, los aumentos en los recursos en educación van subsecuentemente a beneficiar mayoritariamente a estos sectores privados, aumentando aún más sus ganancias y la segregación socio educativa en el sistema chileno.

D. Lo anterior es posible porque las políticas implementadas en los últimos años, principalmente la Gratuidad para la Educación Superior, la Ley de Inclusión y la Nueva Educación Pública, no garantizan el trato preferente del Estado a las instituciones públicas, dejándolas en peores condiciones, permiten y fomentan la proliferación de entidades privadas y no alteran significativamente la estructura de financiamiento de la educación en Chile. Por el contrario, profundizan la carencia de aportes basales a la educación pública y no modifican la posibilidad de lucrar con recursos estatales y entregados por las familias, sino que permiten el lucro a través de nuevos mecanismos.

E. No se han observado cambios relevantes en los últimos años que apunten a un monitoreo transparente y público de los presupuestos, tampoco se ha creado una plataforma donde se realice de manera más democrática y participativa esta fiscalización donde se considere la opinión de los distintos actores del sistema educativo.

\section{PARTICIPACIÓN DE LA SOCIEDAD CIVIL}

A. La participación de la sociedad civil en las políticas educativas es escasa, quedando relegada principalmente a la entrega de petitorios a las autoridades y a ocasionales mesas de trabajo propiciadas por movilizaciones estudiantiles, cuya relevancia es testimonial ya que no son consideradas realmente en la elaboración de las políticas en cuestión. Distinta recepción tienen algunas personas y organizaciones ${ }^{11}$, en especial las ligadas a instituciones privadas, algunas de ellas con claros intereses en el lucro, quienes a cierto nivel logran un diálogo con las autoridades y realizan lobby para la consecución de sus intereses.

\footnotetext{
${ }^{11}$ Ejemplos de dichas organizaciones son: Corporación Emprender, Desafío Levantemos Chile, Educación 2020, Educa UC, Universidad de las Américas, etc. Organizaciones de la sociedad civil generan propuestas para contribuir a mejorar la calidad de la educación inicial, noviembre de 2018. Disponible en: http://centrojusticiaeducacional.cl/organizaciones-de-la-sociedad-civil-generan-propuestas-para-contribuira-mejorar-la-calidad-de-la-educacion-inicial/
} 
B. Desde la sociedad civil han existido análisis y propuestas referentes al sistema educativo y son de larga data, incluso desde antes de las movilizaciones estudiantiles del año 2006. Estas propuestas han incluido aspectos políticos y económicos, muchas de ellas levantadas desde movimientos y organizaciones sociales que han manifestado constantemente su deseo de aportar en la construcción de la educación pública. ${ }^{12}$

C. No obstante lo anterior, la respuesta de los distintos gobiernos ha sido clara; por una parte, no responder directamente a las demandas ciudadanas y sus propuestas terminan siempre consolidando, expandiendo y perfeccionando los pilares fundacionales del sistema educativo chileno implantado durante la dictadura de Augusto Pinochet. Paralelamente, se han ignorado gran parte de las recomendaciones hechas desde la academia y desde mesas técnicas convocadas por el propio MINEDUC ${ }^{13}$. Por otra parte, la respuesta de facto ha consistido en la represión de los movimientos sociales por medio de la criminalización, la utilización de medidas represivas y el uso indiscriminado de la fuerza policial, situación que ha sido denunciada reiteradamente a organismos nacionales e internacionales sin resultados trascendentes y que, al día de hoy, mantiene movilizados a amplios sectores del estudiantado y de profesores (Red Contra la Represión a Estudiantes, 2019). Aprovechamos para denunciar que estas medidas dirigidas por los gobiernos han aumentado durante los últimos años cinco años.

D. En Chile se estableció el "Consejo Nacional para la implementación de la Agenda 2030 para el desarrollo sostenible (ODS)" que se encarga de la organización y promoción de las acciones necesarias para el cumplimiento de los ODS. Se han realizado estudios diagnósticos y se publicó una página web http://www.chileagenda2030.gob.cl, donde aparece información relacionada, así como un monitoreo parcial de los avances del país. No obstante, es necesario señalar que la información presentada formalmente por el gobierno de Chile omite aspectos clave para el logro de los ODS, principalmente evidencia proveniente de investigaciones críticas sobre las políticas implementadas e información proveniente desde la sociedad civil, parte de las cuales se han expuesto en páginas anteriores.

\footnotetext{
12 Una parte de estas propuestas se pueden apreciar en el siguiente libro compilatorio: Foro Chileno por el Derecho a la Educación, 2015. Una década de luchas y propuestas por el derecho a la educación. Disponible en: http://www.opech.cl/wp/wp-content/uploads/2015/08/D\%C3\%A9cada-luchas.pdf

También se recomienda el siguiente artículo ¿Cuánto cuesta la escuela que queremos? Propuesta participativa de financiamiento para la educación pública en Chile. Disponible en http://www.opech.cl/comunicaciones/2014/10/cuadernillo cuanto cuesta escuela queremos.pdf

${ }_{13}$ Como ejemplo de esto, algunas propuestas realizadas por expertos que apuntan a reducir la sobrecarga curricular, sustitución del SIMCE, frenar la escolarización de niños con NEE en escuelas especiales y reconvertir estas, transformar radicalmente el sistema de financiamiento, entre otras. Mesa Técnica de Educación Especial, 2015. Propuestas para avanzar hacia un sistema educativo inclusivo en Chile: un aporte desde la educación especial. Disponible en: https://especial.mineduc.cl/wpcontent/uploads/sites/31/2016/08/INFORMEFINALMESATECNICAEDESPECIAL.pdf
} 


\section{CONCLUSIONES}

A lo largo del presente informe se han recorrido 7 ejes clave en lo que al ODS4 se refiere, exponiendo una serie de antecedentes relativos a los desafíos y obstáculos existentes actualmente en Chile. El lograr la implementación de la Agenda 2030 y el cumplimiento del ODS4 requiere esfuerzos importantes en el país, especialmente la necesidad de transformar radicalmente el sistema educativo actual que se rige por parámetros de mercado y considera a la educación como un bien de consumo, y no como un derecho humano a lo largo de la vida. De otra manera no es factible que el Estado sea garante de una educación inclusiva, equitativa y de calidad y promover oportunidades de aprendizaje para todos las y los ciudadanos.

En conclusión, el informe aquí presentado demuestra que el país debido a la política y la acción de sus gobernantes durante los últimos años, no se acerca al logro del ODS4, incluso en varios de sus ejes se demuestran retrocesos significativos. Por ejemplo, creemos urgente detener cuanto antes el avance de las distintas formas de privatización que hay en el sistema educativo chileno en todos sus niveles, uno de los más segregados y con mayor participación privada en el mundo. Esta es una piedra de tope para la integración y la inclusión educativa en Chile y el desarrollo equitativo de sus niñas y niños.

A lo largo de este documento reafirmamos que la educación pública requiere de manera urgente ser fortalecida desde sus principios fundacionales. Mientras se mantenga o se profundice el actual modelo educativo, los conflictos dentro de las escuelas y las instituciones superiores de educación se profundizarán o en el mejor de los casos se mantendrán. Un sistema educativo que excluya a sus estudiantes no está cumpliendo con el propósito que la sociedad espera de él.

Solicitamos a los organismos internacionales pertinentes a la defensa de la niñez que soliciten información al gobierno de Sebastián Piñera una explicación detallada del por qué niñas y niños de los colegios llamados emblemáticos de Santiago, estén siendo perseguidos y reprimidos dentro de las escuelas violentamente por las fuerzas represivas del estado de Chile, denominadas Fuerzas Especiales de Carabineros. Paralelamente, esperamos que los informes de las organizaciones estudiantiles, de profesores, padres y apoderados sean también considerados por parte de estos organismos internacionales para que tengan una imagen de la verdadera situación de crisis que se vive actualmente en la educación pública en Chile.

Adicionalmente, se vuelve imprescindible, por una parte, el potenciar la investigación crítica y rigurosa sobre el sistema educativo chileno para tener mayor conocimiento y claridad sobre los efectos de las políticas más recientes, el real estado de la inclusión en el país, sobre mecanismos innovadores de privatización y sobre los alcances y consecuencias de las formas instauradas dentro de otros múltiples aspectos del sistema educativo.

Es imperativo contar con una participación abierta y amplia de distintos sectores sociales en la Agenda 2030, y que los movimientos sociales puedan hacerse parte de este proceso, abogando por un rol activo de los organismos internacionales involucrados, más que uno meramente receptivo de la información entregada por el Estado, dando cabida a las voces 
de las diversas minorías y actores sociales invisibilizados que apoyan el trabajo por el cumplimiento del ODS4 y luchan por el Derecho a la Educación a lo largo de toda la vida.

\section{REFERENCIAS BIBLIOGRÁFICAS}

Andrade, E. (17 de junio de 2019). Contra proyecto de subvención: JUNJI anuncia paro nacional desde este martes. Obtenido de Diario Uchile:

https://radio.uchile.cl/2019/06/17/contra-proyecto-de-subvencion-junji-anunciaparo-nacional-desde-este-martes /

Banco Central de Chile. (2018). Cuentas nacionales por sector institucional. Santiago: Banco Central.

CADEM. (2019). El Chile que viene, endeudamiento. Santiago: Encuesta CADEM.

Carrillo, F., Espinoza, S., \& Valenzuela, A. (2018). Mercado laboral y educación en Chile. Principales tendencias y resultados en 2015. Santiago: Comisión Nacional de Productividad.

CERC-MORI. (2019). Barómetro de la Política. Santiago: Mori Chile.

Colegio de Profesores de Chile A.G. (Junio de 2006). La crisis del sistema educativo chileno. Obtenido de OPECH:

http://www.opech.cl/bibliografico/doc_movest/InformeWEB.pdf

Comisión Económica para América Latina y el Caribe. (2019). Panorama Social de América Latina, 2018. Santiago: Naciones Unidas.

Cornejo, R. (2006). El experimento educativo chileno 20 años después: Una mirada crítica a los logros y falencias del sistema escolar. Revista Electrónica Iberoamericana sobre Calidad, Eficacia y Cambio en Educación, 4(1), 118-129.

Diario UChile. (3 de junio de 2019). Organizaciones sociales protestan en contra de la represión estudiantil. Obtenido de Diario UChile:

https://radio.uchile.cl/2019/06/03/organizaciones-sociales-protestan-en-contrade-la-represion-estudiantil/

El Desconcierto. (12 de octubre de 2017). La niñez marcada: Historias de violencia policial contra la infancia mapuche. Obtenido de El Desconcierto: https://www.eldesconcierto.cl/nacional/2017/10/12/la-ninez-marcada-historiasde-violencia-policial-contra-la-infancia-mapuche.html

Encuesta CEP. (2019). Estudio Nacional de Opinión Pública N 83. Santiago: CEP Chile. 
Espacio Vinculante por la Nueva Educación Pública. (2019). La privatización de la educación parvularia. Obtenido de OPECH: http://opech.cl/la-privatizacion-de-laeducacion-parvularia/

Foro Chileno por el Derecho a la Educación. (abril de 2015). Análisis crítico de la ley que regula la admisión de los y las estudiantes, elimina el financiamiento compartido y prohíbe el lucro en establecimientos educacionales que reciben aportes del Estado. Obtenido de OPECH:

http://www.opech.cl/comunicaciones/2015/05/index_19_05_2015_analisis_ley_incl usion.pdf

Foro por el derecho a la educación pública. (Abril de 2019). Incluir y no segregar: El desafío principal del sistema de Educación Pública. Obtenido de OPECH: https://opech.cl/wp-content/uploads/2019/04/FODEP-2019-INCLUIR-Y-NOSEGREGAR.-EL-DESAFIO-PRINCIPAL-DEL-SISTEMA-DE-EDUCACI\%C3\%93NP\%C3\%9ABLICA.pdf

Fundación Nodo XXI. (enero de 2016). La "letra chica" de la gratuidad 2016. Obtenido de Nodo XXI: http://www.nodoxxi.cl/director-de-nodo-xxi-analiza-la-letra-chica-de-lagratuidad-2016/

González, J., \& Parra, D. (2016). Mercantilización de la Educación. Comentario sobre la reforma educativa en Chile 2015. Revista Enfoques Educacionales, 71-89. Obtenido de https://revistas.uchile.cl/index.php/REE/article/view/44633/46650

Inzunza, J., \& Campos-Martínez, J. (2016). El SIMCE en Chile: Historia, Problematización y Resistencia. XI Seminario Internacional de la Red ESTRADO: Movimientos Pedagógicos y Trabajo Docente en tiempos de estandarización. México.

Ministerio de Desarrollo Social. (2018). Síntesis de resultados, encuesta CASEN 2017. Santiago: Ministerio de Desarrollo Social.

MOVILH. (2018). Informe Anual de Derechos Humanos de la Diversidad Sexual y de Género en Chile. Santiago: Hechos.

OECD. (2018). Educación en Chile, Revisión de Políticas Nacionales de Educación. Ciudad de México: OECD Publishing. doi:http://dx.doi.org/10.1787/9789264284425-en

Red Contra la Represión a Estudiantes. (3 de junio de 2019). Declaración «Red Contra la Represión a Estudiantes». iiLas y los estudiantes no están Solas/os!! Obtenido de OPECH: https://opech.cl/declaracion-red-contra-la-represion-a-estudiantes-las-ylos-estudiantes-no-estan-solas-os/

Sáez, B. (29 de marzo de 2019). Contradicciones del desarrollo en Chile. Obtenido de Fundación Sol: https://fundacionsol.cl/blog/presentaciones4/post/contradicciones-del-desarrollo-en-chile-6469 
Revista Enfoques Educacionales - Año 2019 - Vol. 16 No.1: 77-95

Subsecretaría de Educación Parvularia. (2019). Informe de Caracterización de la Educación Parvularia. Santiago: Ministerio de Educación. 